\title{
The Role of Targeted Agents in the Treatment of Metastatic Breast Cancer
}

\author{
Joachim Bischoff Atanas Ignatov \\ Department of Gynecology and Obstetrics, University Hospital Otto-von-Guericke Magdeburg, Germany
}

\section{Key Words}

Breast cancer, metastatic $\cdot$ Anti-angiogenesis .

Antibody · PARP inhibition · Multitargeting

\section{Summary}

To date, blockade of growth factor receptors is the mainstay of targeted therapy in metastatic breast cancer (mBC). Monoclonal antibodies such as trastuzumab and bevacizumab represent the first generation of molecular-based therapies. Both the HER2 inhibitors and the vascular endothelial growth factor (VEGF) antagonists have shown synergism with a broad spectrum of established cytotoxins, thus being approved for first-line treatment of $\mathrm{mBC}$ in combination with taxanes. As a next step, tyrosine kinase inhibitors (TKIs) have been integrated into daily routine as an alternative approach for targeting HER2: The dual HER1/2 inhibitor lapatinib demonstrated activity in trastuzumab-pretreated $\mathrm{mBC}$ patients in combination with capecitabine. Furthermore, chemotherapy-free regimens (trastuzumab or lapatinib plus aromatase inhibitors) have been identified as additional options for hormone receptor (HR)- and HER2-positive patients. Recently published data indicate that a combination of two biologicals such as lapatinib and trastuzumab can be effective as a treatment beyond trastuzumab related progression. Multitarget TKIs have the potential to inhibit several signaling pathways involved in breast cancer-related angiogenesis. Until now, they have failed to show a clear benefit in $\mathrm{MBC}$. On the other hand, poly(ADP-ribose) polymerase (PARP) inhibition, mediated by a new class of small molecules, is an interesting area of investigation. Future directions of research in HER2-positive breast cancer focus on the evaluation of novel antibodies (pertuzumab, T-DM1), and irreversible TKIs (neratinib, BIBW 2992) and inhibitors of HER2-related downstream signaling (mTOR, TORC 1/2, $\mathrm{PI}$ KK/Akt) and of receptor cross-talk (IGFR).

\author{
Schlüsselwörter \\ Mammakarzinom, metastasiertes - Anti-Angiogenese · \\ Antikörper · PARP-Inhibition · Multitargeting
}

\section{Zusammenfassung}

Momentan basiert die zielgerichtete Therapie des metastasierten Mammakarzinoms vor allem auf der Blockade von Wachstumsfaktorrezeptoren. Monoklonale Antikörper wie Trastuzumab und Bevacizumab repräsentieren dabei die erste Generation molekularer Behandlungsansätze. Sowohl der HER2-Inhibitor wie auch der Vascular Endothelial Growth Factor (VEGF)-Antagonist besitzen eine synergistische Wirkung mit einem breiten Spektrum an etablierten Zytostatika. Daraus resultiert unter anderem die Zulassung beider Substanzen in der First-Line-Therapie des Mammakarzinoms in Kombination mit Taxanen. Als weiterer Ansatz zur HER2-gerichteten Therapie haben inzwischen auch Tyrosinkinase-Inhibitoren Eingang in die Behandlungspraxis gefunden. So besitzt der duale HER1/2-Inhibitor Lapatinib einen Stellenwert bei Trastuzumab-vorbehandelten Patientinnen mit HER2-positivem metastasierten Mammakarzinom in Kombination mit Capecitabine. Darüber hinaus stellen chemotherapiefreie Regime (Trastuzumab oder Lapatinib plus Aromataseinhibitoren) eine Option für Hormonrezeptor- und HER-2positive Patientinnen dar. Aktuellen Daten zufolge zeigen Kombinationen zweier Biologicals wie etwa Lapatinib und Trastuzumab eine Effektivität bei einer Progression unter Trastuzumab. Multitarget-Inhibitoren besitzen grundsätzlich das Potenzial, Angiogenesehemmung auf einer breiteren Basis zu betreiben, als dies beim Anti-VEGF-Antikörper Bevacizumab der Fall ist. Bislang ist ihr Stellenwert beim Mammakarzinom allerdings noch unklar. Einen vielversprechenden Ansatz bietet andererseits die Poly(ADPRibose)-Polymerase (PARP)-Inhibition durch eine neue Klasse von "small molecules". Beim HER2-positiven Mammakarzinom stehen derzeit neue Antikörper (Pertuzumab, T-DM1), irreversible TKIs (Neratinib, BIBW 2992) sowie Inhibitoren des Downstream Signalings (mTOR, TORC 1/2, PI3K/Akt) und des Rezeptor-Crosstalks (IGFR) im Fokus der klinischen Forschung.

\begin{tabular}{ll}
\hline KARGER & @ 2010 S. Karger GmbH, Freiburg \\
Fax +497614520714 & Accessible online at: \\
Information@Karger.de & www.karger.com/brc \\
www.karger.com &
\end{tabular}

Dr. med. Joachim Bischoff

Otto-von-Guericke Universität

Medizinische Fakultät, Universitätsfrauenklinik Magdeburg A.ö.R

Gerhart-Hauptmann-Straße 35, 39108 Magdeburg, Germany

Tel. +49 391 67-17301, Fax -17317

joachim.bischoff@med.ovou.de 


\section{Introduction}

Implementation of molecular biological knowledge into the routine of clinical practice has led to therapeutical progress in breast cancer and allowed the identification of new subgroups of tumors. Especially HER2-positive tumors have been treated as a separate collective since the establishment of HER2-targeted therapies. In contrast, HER2-negative carcinomas are a heterogeneous population, including the prognostically favorable luminal subtype with high hormone receptor (HR) expression rate (luminal A) as well as aggressive triple-negative carcinomas.

\section{Anti-Angiogenesis}

Angiogenesis is recognized as one of the critical events required for tumor progression, where cancerous growth is dependent on vascular induction and the development of a neovascular supply. Anti-angiogenic therapy stems from the fundamental concept that tumor growth, invasion and metastatis are angiogenesis dependent. The microvascular endothelial cell recruited by a tumor has become an important second target in cancer therapy. Angiogenesis research plays a key role in oncology since central mediators have been identified in the essential process of tumor development. Pro- and anti-angiogenic factors are involved in complex interaction in order to maintain vascular equilibrium in the physiological state. This equilibrium is jeopardized when a need for increased oxygen arises in consequence of malign growth, which stimulates the generation of hypoxy-inducible factor 1 (HIF-1). Activation of HIF-1 is also involved in the up-regulation of serveral angiogenic factors.

\section{Vascular-Targeted Antibodies}

Each step in the angiogenetic regulatory pathway represents a potential target for therapeutic development. Anti-angiogenic therapy is based on the inactivation of members of the vascular endothelial growth factor receptor (VEGFR) family, resulting in relevant anti-tumor activity. Perhaps the best-validated antiangiogenic approach involves blockade of the vascular endothelial growth factor (VEGF) and its signaling pathway. Indeed, inhibitors that block the VEGF pathway have shown extensive pruning of the rapidly growing tumor vasculature, decreasing microvessel density and normalizing the remaining blood vessels, thereby improving the delivery of chemotherapy. In combination with taxanes, bevacizumab was the first angiogenesis inhibitor to be approved as first-line therapy for HER2negative breast cancer in many countries. The humanized monoclonal antibody binds selectively to the cytokine VEGF-A, the ligand for VEGFR 1 and 2. In combination with paclitaxel ( 36.9 vs. $21.2 \%$ ) as well as with docetaxel (63 vs. $44 \%$ ), bevacizumab significantly increased the response rate in first-line therapy for HER2-negative breast cancer, compared to the re- spective taxane standard therapy. However, prolongation of progression-free survival (PFS) of less than 1 month was significantly shorter in the avastin and docetaxel (AVADO) study, compared to a combination regimen with paclitaxel and bevacizumab (median 11.8 vs. 5.9 months). Nonetheless, in the Eastern Cooperative Oncology Group (ECOG) 2100 study, the near-duplication of PFS had no impact on overall survival [1, 2]. So far, available data from the AVADO study show a 1-year survival rate, favoring the bevacizumab regimen ( 83 vs. $78 \%$ ). The lower bevacizumab dose of $7.5 \mathrm{mg} / \mathrm{kg}$ q3w, e.g. administered in colorectal cancer, was evaluated in this study as well. A trend towards better response and 1-year survival was observed for the higher dosage (15 mg/kg q3w) [3].

Subgroup analyses of both studies revealed that patients receiving prior adjuvant taxane therapy gained a marked benefit from bevacizumab and renewed taxane administration. On the other hand, occasionally observed persistence of taxane-induced polyneuropathy prevented rechallenge of taxanes in the metastatic state.

In this context, the role of taxane-free bevacizumab combinations should be considered. According to an international phase III study (RIBBON-1), improvement of overall response and PFS was achieved by combining the VEGF antibody with capecitabine, antracyclines and taxanes [4].

Administration of angiogenesis inhibitors in further lines remained questionable in the past, based on models considering the limiting role of angiogenesis in late tumor states as well as results from a phase III study with capecitabine and bevacizumab in this setting [5]. The multinational RIBBON-2 study addressed this issue by randomizing almost 700 patients $2: 1$ for chemotherapy with either taxanes $(n=201)$, gemcitabine $(\mathrm{n}=108)$, capecitabine $(\mathrm{n}=97)$ or vinorelbine $(\mathrm{n}=53)$ plus bevacizumab or chemotherapy plus placebo $(n=225)$. After 15 months of follow-up, pooled evaluation of the four chemotherapy cohorts showed a significant reduction of about $22 \%$ (hazard ratio $(\mathrm{HR}) 0.78 ; \mathrm{p}=0.0072$ ) in the progression risk and a 2-month prolongation of PFS compared to chemotherapy alone (7.2 vs. 5.1 months). This was consistent with results from a separate analysis of 3 out of the 4 cohorts: Combining bevacizumab with taxanes, gemcitabine or capecitabine resulted in an equally significant risk reduction for progression. In the vinorelbine cohort, however, the bevacizumab combination did not show any benefit. This might be explained by the small size of the subgroup, including only 23 patients in the placebo arm.

The pooled overall response rate (ORR) increased from $29.6 \%$ in the placebo arm to $39.5 \%$ in the combination arm. At this point, a tendency benefit of bevacizumab was observed in overall survival, achieving a relative reduction in mortality of $10 \%$ (HR $0.90 ; p=0.37$ ). However, the final survival analysis requiring the achievement of $75 \%$ of all results is not yet available [6].

To date, the optimal duration of bevacizumab-based therapy in metastatic breast cancer (mBC) has not yet been de- 
fined. However, there is some evidence that the combination with the respective cytostatic agent should be switched to maintenance therapy with the VEGF antibody after 6-8 cycles, depending on the remission dynamics [7].

\section{Prediction of Response}

Available data show that VEGF is a promising target for targeted therapy of $\mathrm{mBC}$. However, data also indicate limitations in the use of this novel mode of action. Defining a patient group within the HER2-negative collective that benefits from anti-VEGF therapy has failed so far. Identification of valid biological markers remained unsuccessful in spite of extensive research. However, a retrospective analysis from the E2100 study indicated that single nucleotide polymorphisms (SNPs) in VEGF are associated with sensitivity towards bevacizumab. Recent analysis of polymorphisms in the VEGF gene have revealed an association between patients with VEGF-2578 AA and VEGF-1156 AA genotypes and better overall survival versus the other genotypes in the bevacizumab-treated arm. In addition, it was shown that patients with the VEGF-1498 TT and VEGF-634 CC genotypes were largely protected from serious hypertension compared to the other genotypes [8]. Prospective studies are required to confirm these results.

\section{Multitargeted Agents}

Multitargeting addresses the problem of adaptability and redundancy in molecular pathways. This diversity in possible signal transduction routes allows cancer cells to have flexibility in bypassing the inhibited receptor. Despite the positive results seen with VEGF inhibitors, clinical resistance remains an issue as these compounds block only one proangiogenic protein (e.g., VEGF) whereas many human tumors produce multiple proangiogenic proteins, which allows the tumor to evade angiogenic blockade. Therefore, additional targets and pathways are exploited to broaden the anti-angiogenic spectrum. Several other members of the receptor tyrosine kinase (RTK) superfamily besides VEGFR 1-3 are involved as angiogenesis promoters in the development and progression of breast cancer. For example, a higher expression of the platelet-derived growth factor receptor (PDGFR) is associated with higher incidence of metastases, relative resistance against chemotherapy, and shorter survival. In addition, overexpression of the stem cell factor receptor (c-kit) has been demonstrated for breast cancer, preferentially in the triple-negative subtype $[9,10]$.

Sunitinib is a member of this new generation of angiogenesis inhibitors, known to successfully inhibit the entire scope of RTKs. In contrast to bevacizumab, sunitinib antagonizes tyrosin kinase activity at the intracellular receptor domain. Sunitinib is the first multi-tyrosine kinase inhibitor (mTKI) to be tested in a prospective phase III trial against chemother- apy. HER2-negative patients receiving prior anthracycline and taxane therapy up to two regimens in the metastatic state were included in the study. The primary objective was to exceed PFS compared to the control arm receiving capecitabine treatment by at least $33 \%$. However, initial interim analysis revealed that the primary study objective was impossible to achieve. Consequently, the study was prematurely discontinued after 482 patients had been recruited. Sunitinib had proven to be inferior in response as well as PFS [11]. This can be interpreted in different ways:

The biologicals approved for breast cancer treatment so far show moderate activity as single agents. The publication of bevacizumab data has already occasioned a debate on the significance of angiogenesis inhibitors in the later metastatic state. After all, more than $80 \%$ of patients from the sunitinib study had received more than one regimen in the metastatic state.

Results from two randomized phase II trials testing sorafenib, another mTKI in first- and second-line therapy of HER2-negative breast cancer, provides further information regarding the feasibility of mTKI-based combination. The drug profile of sorafenib is similar to that of sunitinib; however, sorafenib has higher affinity to the intracellular raf kinase. Combined with capecitabine, sorafenib significantly improved the primary endpoint, PFS, compared to the respective cytostatic agent alone. However, the experimental regimen was associated with increased non-hematological toxicity. Hand foot syndrome (HFS) was the most relevant adverse event, with grade 3 occurring in $45 \%$ (sorafenib + capecitabine) and 33\% (sorafenib + paclitaxel) of patients, thus surpassing the incidence of skin reactions extensively occurring in poly-chemotherapies or bevacizumab combinations $[12,13]$. In the light of the data reported above, a certain dilemma regarding the administration of mTKIs in breast cancer becomes obvious:

On the one hand, the results indicate that combining multikinase inhibitors with cytostatic agents in further trials may be beneficial. On the other hand, toxicities may be a limiting factor. The evaluation of combining agents with a low risk for overlapping non-hematological toxicities (HFS, diarrhea) should be one objective in future trials with this class of agents.

\section{Poly(ADP-Ribose) Polymerase Inhibition}

As mentioned above, from the current tumor-biological standpoint, HER2-negative breast cancer is heterogeneous. Here, the triple-negative breast cancer (TNBC) subtype remains a challenge, for various reasons:

The aggressive phenotype is associated with a higher risk for early development of distant metastases despite administration of modern adjuvant therapies [14]. While the majority of tumors respond well to the same anthracycline- and taxanebased regimens in the neoadjuvant setting [15], the chemosensitivity of TNBC has yet to be determined. 
The development of targeted therapies was limited by lack of molecular knowledge on triple-negative carcinomas in the past. The finding that these tumors are often characterized by increased poly(ADP-ribose) polymerase-1 (PARP-1) activity enabled further progress in developing targeted therapies [16].

Naturally occurring DNA repair mechanisms are a primary reason that tumors are fundamentally insensitive or become resistant to DNA-damaging therapies. PARP-1, a member of a large family of multifunctional enzymes, plays a key regulatory role in a DNA repair mechanism called base excision repair, which repairs single-strand breaks in DNA [17]. Inhibiting PARP-1 may prevent cancer cells from employing base excision repair to repair damage caused by DNA-damaging therapies. The inhibition of PARPs leads to the accumulation of DNA double-strand breaks at replication forks. This stops DNA replication and ultimately triggers apoptosis. PARP-1 activity may render cancer cells insensitive or resistant to DNA-damaging therapies. Inhibiting PARP-1 may enhance or restore sensitivity to these therapies [18].

\section{BSI-201}

The combination of gemcitabine, carboplatin and the small molecule BSI-201 provides an example for the synergy between DNA-damaging agents and PARP inhibitors. A prospective randomized phase II study was designed on the basis of these data. 62 patients received poly-chemotherapy as described above whereas 61 patients received the experimental triple therapy. PARP-1 expression was verified in the majority of patients. Many patients had received prior anthracycline and taxane therapy. The study featured an extraordinarily high amount of patients with brain metastases (30\%) compared to other studies on $\mathrm{mBC}$. Previous treatment with up to two regimens in the metastatic state was allowed. Primary endpoints were clinical benefit rate and safety [19].

BSI-201 plus gemcitabine/carboplatin improved the clinical benefit rate (62 vs. $21 \%, \mathrm{p}=0.0002)$, objective response rate ( 48 vs. $16 \%, \mathrm{p}=0.0002$ ), median PFS (6.9 vs. 3.3 months, HR $=0.342, \mathrm{p}<0.0001)$, and median overall survival (9.2 vs. 5.7 months, $\mathrm{HR}=0.348, \mathrm{p}=0.0005)$ compared with gemcitabine/ carboplatin alone.

BSI-201 was well tolerated. Gemcitabine- and carboplatinassociated side effects were not enhanced by the agent. No toxicities were reported during the application of BSI-201. Currently, a multinational phase III study with a similar twoarmed design is in progress to confirm these data.

\section{Olaparib}

At the same time, preliminary data from olaparib, another PARP inhibitor being currently evaluated in $\mathrm{mBC}$, were disclosed at the 2009 American Society of Clinical Oncology (ASCO) congress. The patient collective in this trial was restricted to BRCA1/2 mutations because these tumor suppressor proteins are involved in DNA repair in the same way as
PARP-1 and -2, which results in a dysfunctional DNA repair system in BRCA1/2-deficient tumors. In consequence, these tumors are more sensitive towards PARP inhibition in vitro than the wild type [20]. Clinical proof of concept was achieved in a mixed collective of solid tumors. In addition, the optimal dose was established. The following phase II trial enrolled 54 patients who were randomized into two cohorts receiving $400 \mathrm{mg}(\mathrm{n}=27)$ and $100 \mathrm{mg}(\mathrm{n}=27)$, respectively, oral olaparib twice daily. The patients were intensively pretreated. The median number of 3 regimens consisted mainly of anthracyclines and taxanes, combined with platinum derivatives in $20-30 \%$ of cases. $50 \%$ of the patients were triple negative. A response rate of $41 \%$ and a PFS of 5.7 months were achieved with the higher dosage compared to $22 \%$ and 3.8 months with the lower dosage, establishing $400 \mathrm{mg}$ as the daily recommended dose for further evaluation. Olaparib was well tolerated. Grade 3 toxicities were restricted to the occurrence of nausea and fatigue.

In conclusion, PARP inhibitors are promising candidates for establishing the first specific therapy of TNBC in the conceivable future. However, conclusions about the exact significance of this new class of small molecules would be premature. The efficacy of monotherapy versus combination therapy with conventional cytostatic agents remains yet to be compared. Future studies should also evaluate the impact of molecular parameters like PARP expression or BRCA mutations on predicting success with BSI-201 and olaparib treatment [21].

\section{HER2-Positive Breast Cancer}

Trastuzumab as the prototype of a new generation of anti-neoplastic drugs binds to oncogenic RTKs, thus inhibiting tumor-specific signal transduction [22]. Overexpression of human epidermal growth factor receptor 2 (HER2) is of central significance in the pathogenesis of breast cancer, which is consistent with the high efficiency observed with monoclonal HER2 antibodies. In this context, molecular genetic investigations revealed that HER2-positive breast cancer is a well-defined entity featuring a characteristic gene expression profile. Although primarily aggressive, HER2-positive tumors can turn into a prognostically favorable disease when highly efficient trastuzumab treatment is administered. Based on this success, therapeutic strategies have been developed to optimize HER2 targeting and to inhibit HER2-dependent downstream signaling. The therapeutical range includes modified HER2 antibodies (pertuzumab, T-DM1) and small molecules that inhibit several members of the HER family while showing different reversibility in binding to the intracellular receptor domain (lapatinib, neratinib, BIBW 2992). It also includes mammalian target of rapamycin (mTOR) inhibitors that prevent activation of the phosphatidylinositol 3-kinase (PI3K)/ Akt pathway. 
Lapatinib was the first HER2 inhibitor showing activity in

The standard of first-line therapy for HER2-positive breast cancer consists of trastuzumab combined with taxane. This regimen significantly improved response rate, time to progression (TTP) and overall survival in comparison to paclitaxel or docetaxel alone $[23,24]$. In both studies, data were derived exclusively from trastuzumab-naive patients. In current practice, however, HER2 antibody and taxanes are administered during primary treatment in most HER2-positive patients. Despite insufficient evidence, it appears reasonable to re-administer trastuzumab, if necessary, in combination with taxane. A relapse-free interval of 6-12 months should be observed.

The implementation of targeted therapies in breast cancer allows the renunciation of cytostatic drugs to a certain degree. Combinations with endocrine therapies has proved to be a promising approach in this context as cross-talk between estrogen receptor and HER2 signaling is responsible for endocrine resistance in breast tumors with HER2 overexpression. A proof-of-concept study (TAnDEM) showed that combining trastuzumab and anastrozol leads to the duplication of PFS compared to anastrozol alone (2.4 vs. 4.8 months) [25].

The same strategy integrated in a phase III study was conducted to compare lapatinib plus letrozol versus letrozol alone in trastuzumab-naive patients with HR-positive breast cancer. A subgroup of 219 out of 1286 patients had HER2-positive breast cancer. Less than $10 \%$ of patients had received prior anthracyclin and taxane treatment. Previously, there has been limiting experience to employ the dual HER1/2 TKI lapatinib as first-line therapy in a trastuzumab-naive collective. The primary endpoint of the study was achieved by significantly prolonging median PFS from 3.0 to 8.2 months in the combined therapy arm. Significance was also reached in the improvement of the response rate from 15 to $28 \%(\mathrm{p}=0.021)$ as well as the clinical benefit rate (29 vs. $48 \%, \mathrm{p}=0.003)$ [26].

Both studies represent a new and well-tolerated treatment principle that may be employed as first-line therapy in selected cases of HR- and HER2-positive breast cancer. Considering the low efficacy compared to taxane/trastuzumab combinations, indication should be limited to patients with lowrisk metastases.

\section{HER2-Positive mBC-Treatment in Further Lines}

The role of HER2 inhibition as a continuum within different therapy lines for HER2-positive breast cancer in the adjuvant and metastatic state has been generally accepted. Clinical experience with trastuzumab has shown that sensitivity towards the HER2 antibody is maintained in many cases for a very long period compared to cytostatic agents. Despite long-time survival, the issue of resistance against trastuzumab needed to be addressed as well. HER2-positive breast cancer after intensive pretreatment with trastuzumab and other cytostatic regimens:

In a randomized phase III study $(\mathrm{n}=399)$, patients pretreated with anthracycline, taxane and trastuzumab received either lapatinib + capecitabine or capecitabine alone (EGF100151). The majority of patients received several prior regimens in the metastatic setting. Initial interim analysis revealed a significant benefit in the primary endpoint TTP in favor of the combined-treatment arm, which led to the premature discontinuation of the study. A low incidence of brain metastases during the course of disease was noted in the combined-treatment arm [27, 28]. The central nervous system (CNS)-specific antitumor effect of lapatinib was supported by data from a phase II study focusing specifically on a patient collective with brain metastases [29]. Following this evaluation, lapatinib was registered for second- and further-line therapy in HER2-positive breast cancer. Approval was limited to the combinations with capecitabine after previous anthracycline, taxane and trastuzumab treatment. In accordance with a positive opinion from the European Medicines Agency (EMEA), approval is currently extended to first-line therapy in combination with aromatase inhibitors in trastuzumab-naive patients.

\section{Trastuzumab - Treatment beyond Progression}

Continuous anti-HER2 therapy was evaluated in different therapy lines, including maintenance of trastuzumab administration. Different cytostatic drugs are considered for combination in treatment beyond progression (TBP) because of comparatively rapid emergence of resistance. This approach is based on the well-documented synergism between trastuzumab and several established cytostatic drugs for breast cancer $[30,31]$. Pre-clinical studies demonstrated a persisting antiproliferative effect of trastuzumab during the entire exposition period, while discontinuation of the agent instantly triggers tumor growth [32]. This observation was implemented into clinical practice during a prospective multicenter study comparing combined therapy with trastuzumab and capecitabine as second-line therapy versus capecitabine alone. A significant difference was achieved in the primary objective TTP (5.6 vs. 8.2 months), favoring the combined-treatment arm. TBP, therefore, provides a valid alternative to lapatinib and capecitabine. Mature data on overall survival are not available at this time [33]. Drawing definitive conclusions from comparing data of both studies is impossible because both collectives received different prior treatment (GBG 26 second-line patients, EGF100151 second-line/heavily pretreated patients).

Combining lapatinib and trastuzumab $(\mathrm{L}+\mathrm{T})$ provides another option in this setting. 291 women were enrolled in a randomized phase II trial (EGF104900), comparing L + T against lapatinib (L) alone in the standard arm [34]. A second updated survival analysis, conducted when $75 \%$ of all results 
were achieved, revealed a 4.5-month prolongation of survival (14 vs. 9.5 months) in favor of the combination (HR 0.74, p = 0.026). Patients were heavily pretreated, receiving a mean of 4 and 5 regimens, respectively. There was a very high incidence of brain metastases $(16 / 20 \%)$. Crossover of patients in the lapatinib-treated arm was extraordinary high as well (52\%). In both study arms, striking overall survival was not achieved on the basis of relevant response rates $(10.3 \% \mathrm{~L}+\mathrm{T}$ vs. $6.9 \% \mathrm{~L}$; $\mathrm{p}=0.46)$, which may be indicative of the mode of action of biologicals as non-cytotoxic agents [35]. In summary, the present study may be viewed as pilot study for many reasons:

Heavily pretreated patients have hardly been investigated in prospective studies on breast cancer. In this setting, no survival benefit has been demonstrated by any regimen so far. The randomized study presents the first data on combining TKIs with an antibody in breast cancer. In contrast, the principle of dual HER2 inhibition is being investigated in the combination of trastuzumab and other HER2 inhibitors apart from lapatinib.

\section{Pertuzumab}

In vitro and in vivo experiments have demonstrated that HER2-containing heterodimers elicit greater mitogenic responses than HER2 homodimers. Thus, ligand-induced activation of either HER1 or HER3, with subsequent formation of heterodimers with HER2, may play an important role in resistance to conventional HER2 inhibitors.

Pertuzumab, a novel monoclonal antibody, is a HER2 dimerization inhibitor that binds to a different epitope on the HER2 protein than trastuzumab and inhibits HER2 dimer formation with other HER family members such as HER3 and HER1 [36].

The single-agent activity of pertuzumab in HER2-positive $\mathrm{mBC}$ progressing on trastuzumab was modest (ORR 7\%; $\mathrm{n}=$ 29) [37].

Consistent with their distinct modes of action on HER2 signaling disruption, preclinical studies evaluating pertuzumab in combination with trastuzumab have demonstrated promising therapeutic efficacy. In 66 efficacy-evaluable patients included in a prospective phase II trial, the ORR based on investigator assessment was 24\% (11 partial remission (PR), 5 complete remission (CR)), and the median PFS was 24 weeks. Stable disease lasting at least 8 cycles of treatment ( $~ 6$ months) was observed in $26 \%$ of the treated patients. This drug was generally well tolerated. No relevant cardiac toxicity was observed [38].

\section{mTOR Inhibitors}

Combining trastuzumab with $m$ TOR inhibitors provides another variation of the TBP concept.
Trastuzumab resistance is linked to an increased downstream signaling like PI3K/Akt activation, mediated by PI3K mutations or phosphatase and tensin homolog (PTEN) loss [39].

In vitro studies indicated that everolimus (RAD001) restores trastuzumab sensitivity to PTEN-deficient breast cancer cells, suggesting that mTOR inhibition may rescue trastuzumab resistance caused by PTEN loss.

Everolimus, a rapamycin ester analog, acts by selectively inhibiting mTOR, an intracellular serine/threonine protein kinase, and is involved in the regulation of a wide range of growth-related functions and neoplastic cells [40].

A phase I study (CRAD001J2102) evaluated daily and weekly administration of everolimus combined with weekly trastuzumab and vinorelbine $\left(25 \mathrm{mg} / \mathrm{m}^{2}\right.$ day 1 and $\left.8 \mathrm{q} 3 \mathrm{w}\right)$ in patients with HER2-overexpressing $\mathrm{mBC}$.

The trial recruited a total of 50 patients; the median number of prior antineoplastic regimens was 5 (range: 1-10) and the median number of prior chemotherapy regimens for metastatic disease was 2 (range: 0-6). All patients were resistant to trastuzumab; all except 1 received prior taxanes $(46 \%$ of them considered taxane resistant) and $91 \%$ received prior anthracyclines. $10(20 \%)$ patients were pretreated and considered resistant to lapatinib-based therapy.

The main toxicities included: grade 3-4 neutropenia, grade 2-3 stomatitis and fatigue.

Among the 44 evaluable patients for efficacy, $1 \mathrm{CR}$ and 7 PRs have been reported, with an ORR of $18 \%$. An additional 27 patients $(61 \%)$ had disease stabilization.

In the 5-mg daily cohort $(\mathrm{n}=25)$, the median TTPs in the patients who had received $\leq 1$ (11 patients) or $\geq 2$ (14 patients, range 2-6) previous chemotherapy lines for metastatic disease were 43 and 29 weeks, respectively.

The $5 \mathrm{mg}$ daily schedule was selected as the recommended dose in combination with trastuzumab and vinorelbine for further development [41].

\section{Other HER2-Directed Agents}

\section{Trastuzumab-DM1}

Direct covalent coupling of cytotoxic agents to monoclonal antibodies is an alternative to naked antibody-targeted therapy. Currently, only one such conjugate, anti-CD33 conjugated to calicheamicin (gemtuzumab ozogamicin or mylotarg), has been approved for the treatment of relapsed acute myeloid leukemia. Trastuzumab-DM1 (T-DM1) is a first-in-class HER2 antibody-drug conjugate specifically designed for the treatment of HER2-positive breast cancer. T-DM1 is designed to combine trastuzumab's HER2-blocking activity with targeted delivery of the highly potent antimicrotubule agent maytansine derivative (DM1) to HER2-expressig breast cancer cells.

T-DM1 is composed of three constituents: trastuzumab, the linker molecule succinimidyl-4-(N-maleimidomethyl (SMCC), and the cytotoxic agent DM1. 
T-DM1 binds to HER2 with an affinity similar to that of trastuzumab; this binding is required for T-DM1 antitumor activity. The stable linker MCC allows the release of DM1 only when trastuzumab binds to surface HER2 and is internalized, where DM1 is released following lysosomal degradation of T-DM1. Consequently, systemic exposure to free DM1 is minimized while exposure of HER2+ tumors to DM1 is maximized [42].

This is another substance that has shown promising activity in lapatinib-pretreated patients. In the last year, preliminary results from a single-arm phase II trial have been presented. 112 trastuzumab-refractory patients were treated with a median of 3 prior chemotherapy regimens, $55 \%$ of them also with lapatinib after trastuzumab failure. With a median follow-up of 4.4 months, the objective response rate was $39.3 \%$, and $38.3 \%$ for lapatinib-pretreated patients. Thrombocytopenia is the key toxicity (7.1\% grade $3-4)$ [43].

\section{Neratinib}

Lapatinib represents the first-in-class reversible HER1/2 TKI registered for the treatment of HER2 positive $\mathrm{mBC}$.

The strategies of irreversible inhibition are recurrent themes in the development of the second generation of TKIs, of wich neratinib is an example. Irreversible inhibitors can permanently eliminate kinase activity until a new receptor is synthesized. Thus, these agents can provide prolonged suppression of the target, which is probably necessary to achieve maximal antitumor activity.
Moreover, neratinib is a novel TKI that blocks signal transduction through a broad spectrum of 3 receptors, erbB-1, erbB-2 and erbB-4 [44, 45].

Recently, a phase II trial has been conducted, evaluating the role of neratinib in the monotherapy of HER2-positive $\mathrm{mBC}$. Data were available for 136 patients. 68 subjects $(50 \%)$, 34 in each of the 2 treatment groups, had received between 2 and 3 prior cytotoxic regimens; 40 subjects (29\%), 24 (36\%) in treatment group A and $16(23 \%)$ in treatment group B (trastuzumab-naive patients), had received $>3$ prior cytotoxic regimens. $89 \%$ of the subjects in treatment group A had received 1-3 trastuzumab-based regimens in the metastatic setting; $26 \%$ of these subjects had received trastuzumab in the neoadjuvant or adjuvant setting.

The ORR was $24 \%$ for subjects in treatment group A and $56 \%$ for subjects in treatment group B. A PR was reported for 53 evaluable subjects. The 16 -week PFS rate was 59\% and $78 \%$ and the median PFS was 22 weeks, with data censored for 23 subjects, and 40 weeks, with data censored for 32 subjects, for subjects in treatment groups A and B, respectively. Diarrhea (90\% of subjects), which was reversible and generally manageable by supportive care, temporary discontinuation of treatment, and dose reduction regardless of toxicity grade were the most commonly reported side effects [46].

These data suggest that neratinib has a single-agent activity comparable to lapatinib plus capecitabine in trastuzumab-pretreated $\mathrm{mBC}$ patients. These results need to be confirmed by large-scale randomized trials.

\section{References}

1 Miller K, Wang M, Gralow J, Dickler M, Cobleigh M, Perez EA, Shenkier T, Cella D, Davidson NE: Paclitaxel plus bevacizumab versus paclitaxel alone for metastatic breast cancer. N Engl J Med 2007:357:2666-2676.

2 Gray R, Bhattacharya S, Bowden C, Miller K, Comis RL: Independent review of E2100: a phase III trial of bevacizumab plus paclitaxel versus paclitaxel in women with metastatic breast cancer. J Clin Oncol 2009;27:4966-4972.

3 Miles D, Chan A, Romieu G, Dirix LY, Cortes J, Pivot X, Tomczak P, Taran T, Harbeck N, Steger GG: Randomized, double-blind, placebocontrolled, phase III study of bevacizumab with docetaxel or docetaxel with placebo as first-line therapy for patients with locally recurrent or metastatic breast cancer (MBC): AVADO. J Clin Oncol 2008;26:abstr 1011.

4 Robert NJ, Dieras V, Glaspy J, Brufsky A, Bondarenko I, Lipatov O, Perez E, Yardley D, Zhou X Phan S: RIBBON-1: Randomized, double-blind, placebo-controlled, phase III trial of chemotherapy with or without bevacizumab (B) for first-line treatment of HER2-negative locally recurrent or metastatic breast cancer (MBC). J Clin Oncol 2009;27:abstr 1005.
5 Miller KD, Chap LI, Holmes FA, Cobleigh MA, Marcom PK, Fehrenbacher L, Dickler M, Overmoyer BA, Reimann JD, Sing AP, Langmuir V, Rugo HS: Randomized phase III trial of capecitabine compared with bevacizumab plus capecitabine in patients with previously treated metastatic breast cancer. J Clin Oncol 2005;23:792-799.

6 Brusky J, et al.: RIBBON-2: A randomized, doubleblind, placebo-controlled, phase III trial evaluating the efficacy and safety of bevacizumab in combination with chemotherapy for second-line treatment of HER2-negative metastatic breast cancer. Cancer Res 2009;69(suppl 24):abstr 42.

7 Fumoleau P, Greil R, Rayson D, Müller V, Barni S, Aleknavicius E, Tellez E, Wilson C, Miles D: Bevacizumab (BV) maintenance therapy significantly delays disease progression (PD) or death compared with placebo (PL) in the AVADO trial $(\mathrm{BV}+$ docetaxel (D) vs. D + PL in 1st-line HER2negative locally recurrent (LR) or metastatic breast cancer (MBC)). Clin Cancer Res 2008;69:abstr 903.

8 Schneider BP, et al.: Association of vascular endothelial growth factor and vascular endothelial growth factor receptor-2 genetic polymorphisms with outcome in a trial of paclitaxel compared with paclitaxel plus bevacizumab in advanced breast cancer: ECOG 2100. J Clin Oncol 2008;26:46724678 .
9 Seymour L, Dajee D, Bezwoda WR: Tissue platelet derived-growth factor (PDGFR) predicts for shortened survival and treatment failure in advanced breast cancer. Breast Cancer Res Treat 1993;26:247-252.

10 Goswarni S, Sahai E, Wyckoff JB, et al.: Macrophages promote the invasion of breast carcinoma cells via a colony-stimulating factor-1/epidermal growth factor paracrine loop. Cancer Res 2005;65:5278-5283.

11 Barrios C, Liu M, Lee S, Vanlemmens L, Ferrero J, Tabei T, Pivot X, Iwata H, Aogi K, Brickman M, Zhang K, Kern K, Martin M: Phase III randomized trial of sunitinib (SU) vs. capecitabine (C) in patients (Pts) with previously treated HER2negative advanced breast cancer (ABC). Cancer Res 2009:69(suppl 24):abstr 46.

12 Gradishar WJ, Kaklamani V, Sahoo TP, Lokanatha D, Raina V, Boudarde S, Jain M, Schwarztberg L: A double-blind, randomized phase $2 \mathrm{~b}$ study evaluating the efficacy and safety of sorafenib compared to placebo when administrated in combination with paclitaxel in patients with locally recurrent or metastatic breast cancer. San Antonio Breast Cancer Symposium 2009, oral presentation. 
13 Baselga J, Roché H, Costa F, Segalla JGM, Pinczowski H, Ciruelos EM, Cabral Filho S, Gomez P, Van Eyll B, on behalf of the SOLTI-0701 study investigators: SOLTI-0701: A multinational doubleblind, randomized phase $2 \mathrm{~b}$ study evaluating the efficacy and safety of sorafenib compared to placebo in combination with capecitabine in patients with locally advanced or metastatic breast cancer. San Antonio Breast Cancer Symposium 2009, oral presentation.

14 Nofech-Mozes S, Trudeau M, Kahn H, et al.: Patterns of recurrence in the basal and non-basal subtypes of triple-negtive breast cancer. Breast Cancer Res Treat 2009;118:131-137.

15 Liedtke C, Mazouni C, Hess KR, et al.: Response to neoadjuvant therapy and long-term survival in patients with triple-negative breast cancer. J Clin Oncol 2008;26:1275-1281.

16 Carey LA, Dees EC, Sawyer L, et al.: The triple negative paradox: primary tumor chemosensitivitiy of breast cancer subtypes. Clin Cancer Res 2007;13: 2329-2334.

17 Amé JC, Spenlehauer C, de Murcia G: The PARP superfamily. Bioessays 2004;26:882-893.

18 Lewis C, Low JA: Clinical poly(ADP-ribose) polymerase inhibitors for the treatment of cancer. Curr Opin Investig Drugs 2007;8:1051-1056.

19 O'Shaughnessy J, et al.: Efficacy of BSI-201, a poly(ADP-ribose) polymerase-1 (PARP1) inhibitor, in combination with gemcitabine/carboplation $(\mathrm{G} / \mathrm{C})$ in patients with metastatic triple negative breast cancer (TNBC): results of a randomized phase II trial. J Clin Oncol 2009;27(suppl 18):793s, abstr 3 .

20 Fong PC, Boss DS, Yap TA, Tutt A, Wu P, Mergui-Roelvink M, Mortimer P, Swaisland H, Lau A, O'Connor MJ, Ashworth A, Carmichael J, Kaye SB, Schellens JHM, de Bono JS: Inhibition of poly(ADP-ribose) polymerase in tumors from BRCA mutation carriers. N Engl J Med 2009;361: 123-134.

21 Tutt A, Robson M, Garber JE, et al.: Phase II trial of the oral PARP inhibitor olaparib in BRCAdeficient advanced breast cancer. J Clin Oncol 2009;27(suppl 18):abstr CRA501.

22 Hudis CA: Trastuzumab - mechanism of action and use in clinical practice. N Engl J Med 2007;357: 39-51.

23 Slamon DJ, Leyland-Jones B, Shak S, Fuchs H, Paton V, Bajamonde A, Fleming T, Eiermann W, Wolter J, Pegram M, Baselga J, Norton L: Use of chemotherapy plus a monoclonal antibody against HER2 for metastatic breast cancer that overexpresses HER2. N Engl J Med 2001;344:783-792.

24 Marty M, Cognetti F, Maraninchi D, Snyder R, Mauriac L, Tubiana-Hulin M, Chan S, Grimes D, Anton A, Lluch A, Kennedy J, O'Byrne K, Conte P, Green M, Ward C, Mayne K, Extra JM: Randomized phase II trial of the efficacy and safety of trastuzumab combined with docetaxel in patients with human epidermal growth factor receptor 2-positive metastatic breast cancer administered as first-line treatment: the M77001 study group. J Clin Oncol 2005;23:4265-4274.

-25 Kaufman B, Mackey JR, Clemens MR, Bapsy PP, Vaid A, Wardley A, Tjulandin S, Jahn M, Lehle M, Feyereislova A, Revil C, Jones A: Trastuzumab plus anastrozole versus anastrozole alone for the treatment of postmenopausal women with human epidermal growth factor receptor 2-positive, hormone receptor-positive metastatic breast cancer: results from the randomized phase III TAnDEM study. J Clin Oncol 2009;27:5529-5537.
26 Johnston S, Pippen J Jr, Pivot X, Lichinitser M, Sadeghi S, Dieras V, Gomez HL, Romieu G, Manikhas A, Kennedy MJ, Press MF, Maltzman J, Florance A, O'Rourke L, Oliva C, Stein S, Pegram M: Lapatinib combined with letrozole versus letrozole and placebo as first-line therapy for postmenopausal hormone receptor-positive metastatic breast cancer. J Clin Oncol 2009;27:5538-5546.

27 Geyer CE, Forster J, Lindquist D, et al.: Lapatinib plus capecitabine for HER2-positive advanced breast cancer. N Engl J Med 2006;355:2733-2743.

28 Cameron D, Casey M, Press M, Lindquist D, et al.: A phase III randomized comparison of lapatinib plus capecitabine versus capecitabine alone in women with advanced breast cancer that has progressed on trastuzumab: updated efficacy and biomarker analyses. Breast Cancer Res Treat 2008; 112:533-543.

29 Lin NU, Dieras V, Devchand P, Lossignol D, Christodoulou C, Stemmler HJ, Roche H, Liu MC, Greil R, Ciruelos E, Loibl S, Gori S, Wardley A, Yardley D, Brufsky A, Blum JL, Rubin SD, Dharan B, Steplewski K, Zembryki D, Oliva C, Roychowdhury D, Paoletti P, Winer EP: Multicenter phase II study of lapatinib in patients with brain metastases from HER2-positive breast cancer. Clin Cancer Res 2009;15:1452-1459.

30 Fountzilas G, Razis E, Tsavdaridis D, Karina M, Labropoulos S, Christodoulou C, Mavroudis D, Gogas H, Georgoulias V, Skarlos D: Continuation of trastuzumab beyond disease progression is feasible and safe in patients with metastatic breast cancer: a retrospective analysis of 80 cases by the Hellenic Cooperative Oncology Group. Clin Breast Cancer 2003:4:120-125.

31 Montemurro F, Donadio M, Clavarezza M, Redana S, Jacomuzzi ME, Valabrega G, Danese S, Vietti-Ramus G, Durando A, Venturini M, Aglietta M: Outcome of patients with HER2-positive advanced breast cancer progressing during trastuzumab based therapy. Oncologist 2006;11:318-324.

32 Pegram MD, Konecny GE, O'Callaghan C, et al.: Rational combinations of trastuzumab with chemotherapeutic drugs used in the treatment of breast cancer. J Natl Cancer Inst 2004;96:739-749.

33 von Minckwitz G, du Bois A, Schmidt M, Maass N, Cufer T, de Jongh FE, Maartense E, Zielinski C, Kaufmann M, Bauer W, Baumann KH, Clemens MR, Duerr R, Uleer C, Andersson M, Stein RC, Nekljudova V, Loibl S: Trastuzumab beyond progression in human epidermal growth factor receptor 2-positive advanced breast cancer: a German Breast Group 26/Breast International Group 03-05 study. J Clin Oncol 2009;27:1999-2006.

34 Blackwell KL, Burstein HJ, Storniolo AM, Rugo H, Sledge G, Koehler M, Ellis C, Casey M, Vukelja S, Bischoff J, Baselga J, O'Shaughnessy J: Randomized study of lapatinib alone or in combination with trastuzumab in women with erbB2-positive, trastuzumab-refractory metastatic breast cancer. J Clin Oncol 2010;28:1124-1130.
35 Blackwell KL, et al.: A randomized study of lapatinib (Tykerb ${ }^{\circledast}$ ) in combination with trastuzumab versus lapatinib monotherapy in heavily pretreated HER2+ metastatic breast cancer progressing on trastuzumab therapy. Cancer Res 2009;69(suppl): abstr 61.

36 Franklin MC, Carey KD, Vajdos FF, Leahy DJ, de Vos AM, Sliwkowski MX: Insights into ErbB signaling from the structure of the ErbB2-pertuzumab complex. Cancer Cell 2004;5:317-328.

37 Cortés J, Baselga J, Petrella T, et al.: Pertuzumab monotherapy following trastuzumab-based treatment: activity and tolerability in patients with advanced HER2 positive breast cancer. Proc Am Soc Clin Oncol 2009; abstr 1022

38 Baselga J, Gelmon KA, Verma S, Wardley A, Conte PF, Miles D, Bianchi G, Cortes J, McNally VA, Ross GA, Fumoleau P, Gianni L: Phase II trial of pertuzumab and trastuzumab in patients with human epidermal growth factor receptor 2-positive metastatic breast cancer that progressed during prior trastuzumab therapy. J Clin Oncol 2010; 28:1138-1144.

39 Berns K, et al.: A functional genetic approach identifies the PI3K pathway as a major determinant of trastuzumab resistance in breast cancer. Cancer Cell 2007;12:395-402.

40 Lu C-H, Wyszomierski SL, Tseng LM, et al.: Preclinical testing of clinically applicable strategies for overcoming trastuzumab resistance caused by PTEN deficiency. Clin Cancer Res 2007;13:56615662

41 Fasolo A, Gianni L, Rorive A, et al.: Multicenter phase I clinical trial of daily and weekly RAD001 (everolimus) in combination with vinorelbine and trastuzumab in patients with HER-2 overexpressing metastatic breast cancer with prior resistance to trastuzumab. Cancer Res 2009;69:abstr 406.

42 Gail D, Phillips L, Li G, Dugger DL, Crocker LM, Parsons KL, Mai E, Blättler WA, Lambert JM, Chari RVJ, Lutz RJ, Wong WLT, Jacobson FS, Koeppen H, Schwall RH, Kenkare-Mitra SR, Spencer SD, Sliwkowski MX: Targeting HER2-positive breast cancer with trastuzumab-DM1, an antibodycytotoxic drug conjugate. Cancer Res 2008;68:92809290.

43 Vogel CL, Burris HA, Limentani S, et al.: A phase II study of trastuzumab-DM1 (T-DM1), a HER2 antibody-drug conjugate (ADC), in patients (pts) with HER2 positive MBC: final results. Proc Am Soc Clin Oncol 2009; abstr 1017.

44 Rabindran SK, Discafani CM, Rosfjord EC, et al. Antitumor activity of HKI-272, an orally active, irreversible inhibitor of the HER-2 tyrosine kinase. Cancer Res 2004;64:3958-3965.

45 Wong KK, Francasso PM, Bukowski RM, et al. A phase I study with neratinib (HKI-272), an irreversible pan erbB receptor tyrosine kinase inhibitor, in patients with solid tumors. Clin Cancer Res 2009;15:2552-2558.

46 Burstein HJ, Sun Y, Tan AR, et al.: Neratinib (HKI-272), an irreversible pan erbB receptor tyrosin kinase inhibitor: phase 2 results in patients with advanced HER2+ breast cancer. San Antonio Breast Cancer Symposium 2008;abstr 37. 